\title{
Demonstration of the Applicability of Nondestructive Microwave Testing to the Long-Range Inspection of Inner-Surface Cracks in Tubes
}

\author{
Kota Sasaki ${ }^{* 1}$, Takuya Katagiri*1 ${ }^{*}$, Noritaka Yusa ${ }^{* 2}$ and Hidetoshi Hashizume \\ Department of Quantum Science and Energy Engineering, Graduate School of Engineering, Tohoku University, Sendai 980-8579, Japan
}

This study evaluated the applicability of nondestructive microwave testing method for the long-range detection of inner-surface cracks in metallic tubes. Two seamless straight tubes $7 \mathrm{~m}$ in length and $23.0 \mathrm{~mm}$ in inner diameter were prepared, and artificial circumferential slits penetrating the tube walls were introduced. A probe to propagate microwave inside the tube, designed based on three-dimensional finite element simulations, was attached to an end of the tube, and the reflections of the microwave propagating inside the tube were measured using a network analyzer. The experiments confirmed clear reflections due to the slits situated $6 \mathrm{~m}$ away from the probe. Furthermore, imposing a signal processing method to compensate for the dispersion of the microwave clarified the reflections and enabled the slit to be localized quantitatively from the time-of-flight of the reflections. [doi:10.2320/matertrans.M2017008]

(Received January 10, 2017; Accepted February 1, 2017; Published February 24, 2017)

Keywords: electromagnetic nondestructive evaluation, time of flight, localization, signal processing, finite element simulation

\section{Introduction}

A nondestructive microwave testing method has been proposed for the rapid and long-range inspection of degradation of tubes ${ }^{1-5)}$, which would reduce the costs of inspecting long piping systems in complex structures such as power plants ${ }^{6)}$. The basic principle of the method is to propagate microwaves in a tube and detect the reflections caused by anomalies. Because microwaves propagate in a metallic tube with little attenuation, this method would enable quick inspection of the inner surfaces of long tubes without moving the probe emitting the microwaves.

Most previous studies concerning the development of the nondestructive microwave testing have targeted wall thinning inside a tube, and confirmed that the method can clearly detect wall thinning even when the profile of wall thinning is rather complicated ${ }^{7}$. The shape of the probe emitting the microwave affects the microwave mode, which is one of the most dominant factors affecting the signal-to-noise ratio ${ }^{8)}$. Another study has demonstrated that artificial wall thinning is detectable approximately $25 \mathrm{~m}$ from the probe ${ }^{9)}$. In contrast, little previous work has applied the method to crack detection, even though cracks are among the most important degradations. Several studies have reported the use of microwaves to detect artificial slits that simulate cracks $^{1,2)}$ but the reported signals were unclear. Additionally, the tubes used in their experiments were limited to $1-2 \mathrm{~m}$ in length, which is insufficient to determine the applicability of the method to the inspection of long tubes.

Therefore, the present study evaluated the applicability of the nondestructive microwave testing for crack detection using straight metallic tubes $7 \mathrm{~m}$ in length. Seamless tubes were used because an earlier study found that flange connections, which connected many short tubes to simulate a long straight tube, caused reflections comparable to those due to a $\operatorname{crack}^{9)}$.

\section{Materials and Methods}

\subsection{Specimens}

Two tubes $7 \mathrm{~m}$ in length were prepared using modified

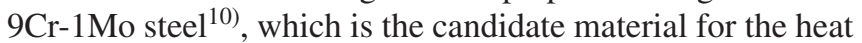
exchanger tubes of the Japan Sodium-cooled Fast Reactor ${ }^{11)}$. The Japan-Sodium-cooled Fast Reactor will employ straighttube heat exchangers containing approximately 4,000 heat exchanger tubes per unit, thus requiring the development of improved tube inspection techniques. The inner and outer diameters of the tubes are 23.0 and $25.4 \mathrm{~mm}$, respectively. Figure 1 shows the flaws introduced into the tubes. The flaws were fabricated by partially cutting the outer surface of the tubes circumferentially using a hacksaw. The resulting flaws penetrated the tube wall with an opening of approximately $1.2 \mathrm{~mm}$. Table 1 provides the specification of the flaws. Measurements were first conducted before flaw fabrication, and then flaws with the circumferential lengths of Length1 were introduced. After the signals due to the flaws were gathered, the flaws were deepened to the circumferential lengths of Length2. The measurements also evaluated the effects of con-

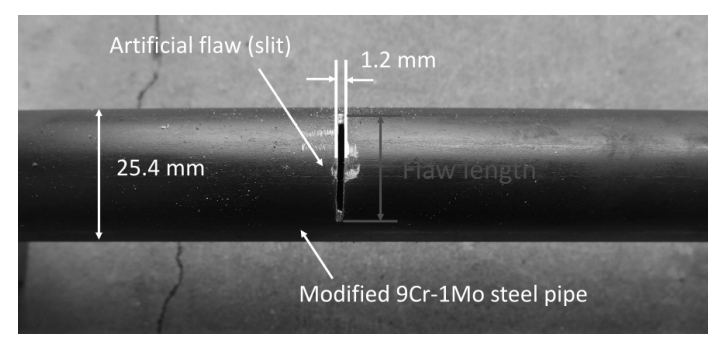

Fig. 1 A tube with a flaw prepared in this study.

Table 1 Specification of flaws fabricated in the tubes.

\begin{tabular}{cccc}
\hline Tube ID & Location from an end, $\mathrm{m}$ & Length1, mm & Length2, mm \\
\hline 1 & 1.0 & 14.65 & 23.90 \\
\hline 2 & 1.5 & 15.30 & 24.80 \\
\hline
\end{tabular}

\footnotetext{
${ }^{* 1}$ Graduate Student, Tohoku University

${ }^{* 2}$ Corresponding author, E-mail: noritaka.yusa@qse.tohoku.ac.jp
} 
ditions at the end of the tube opposite to the probe, which were either open to air or closed by a metallic plate approximately $1 \mathrm{~cm}$ in thickness. The cut-off frequencies of the microwaves propagating inside the tube in axisymmetric modes are listed in Table 2.

\subsection{Experimental setup}

Figure 2 shows the experiments for measuring reflections due to the flaws. Microwaves were generated by a network analyzer (Agilent Technology, E8363B) and propagated in a flexible cable (Junkosha, MWX051) in the TEM mode. The microwaves were emitted inside the tube through the microwave probe that was connected to the flexible cable using a connector (Anritsu, K101F-R) and attached at the end of the tube. The reflections of the microwaves were measured by the

Table 2 Cut-off frequencies of microwave.

\begin{tabular}{ccc}
\hline & $\mathrm{TM}_{0 \mathrm{n}}$ & $\mathrm{TE}_{\text {0n }}$ \\
\hline $\mathrm{n}=1$ & 9.99 & 15.91 \\
\hline $\mathrm{n}=2$ & 22.92 & 29.13 \\
\hline $\mathrm{n}=3$ & 35.93 & 42.24 \\
\hline $\mathrm{n}=4$ & 48.96 & 55.32 \\
\hline & & unit: $\mathrm{GHz}$
\end{tabular}

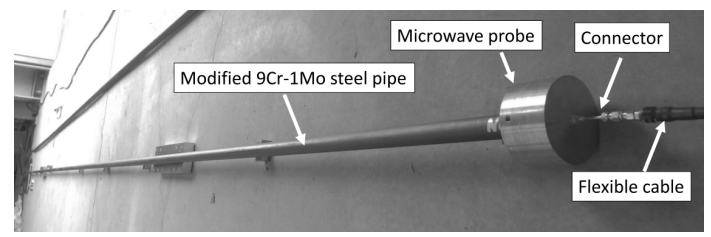

Fig. 2 Appearance of experiments.
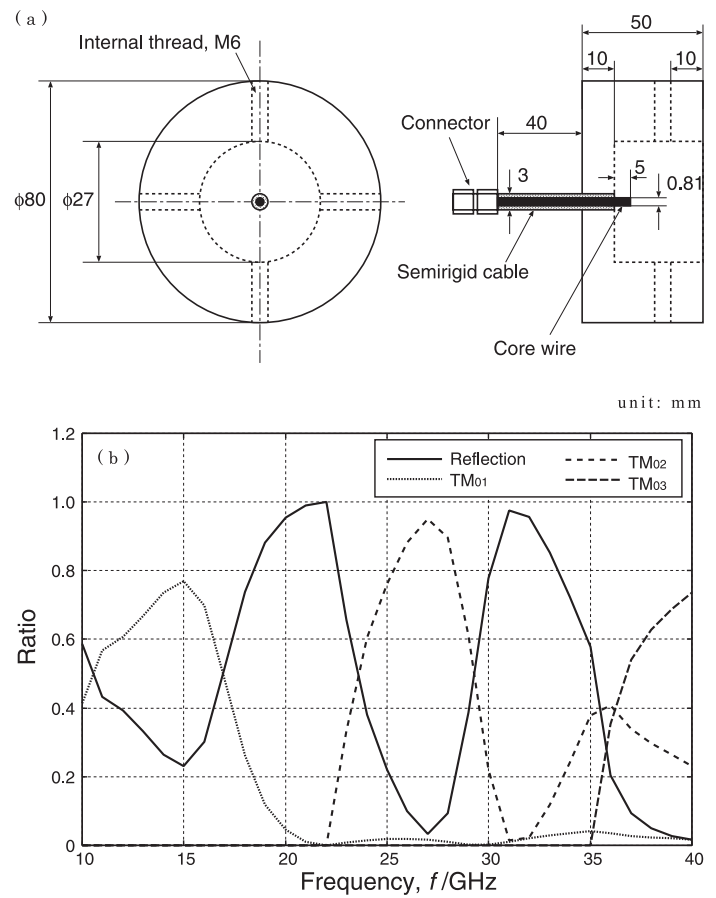

Fig. 3 Microwave probe used in this study to propagate microwave inside a tube. (a) Dimensions of the probe, (b) Characteristics of the probe. network analyzer in the frequency domain at 12,801 equally spaced frequencies ranging from 10 to $40 \mathrm{GHz}$ with an average of 30 measurements. The measured signals were transformed using the inverse Fourier transform with the Kaiser window function $(n=6)$ to obtain signals in the time domain, which confirmed that the reflections occurred when the network analyzer generated the microwave as a pulse.

\subsection{Microwave probe}

Figure 3(a) shows the probe used to emit microwaves inside the tube, which was designed based on three-dimensional finite element simulations using Comsol Multiphysics v.5.0 and its RF module according to the method and principle presented in the reference ${ }^{8)}$. The probe was made of brass with a semirigid cable (Anritsu, K118) situated at its center; the core wire of the semirigid cable was designed to be extended by $5 \mathrm{~mm}$ inside the tube. The four internal threads were used to fix the probe to the tube end.

Figure 3(b) presents the characteristics of the probe, namely the ratios of energies reflected at the probe and propagating
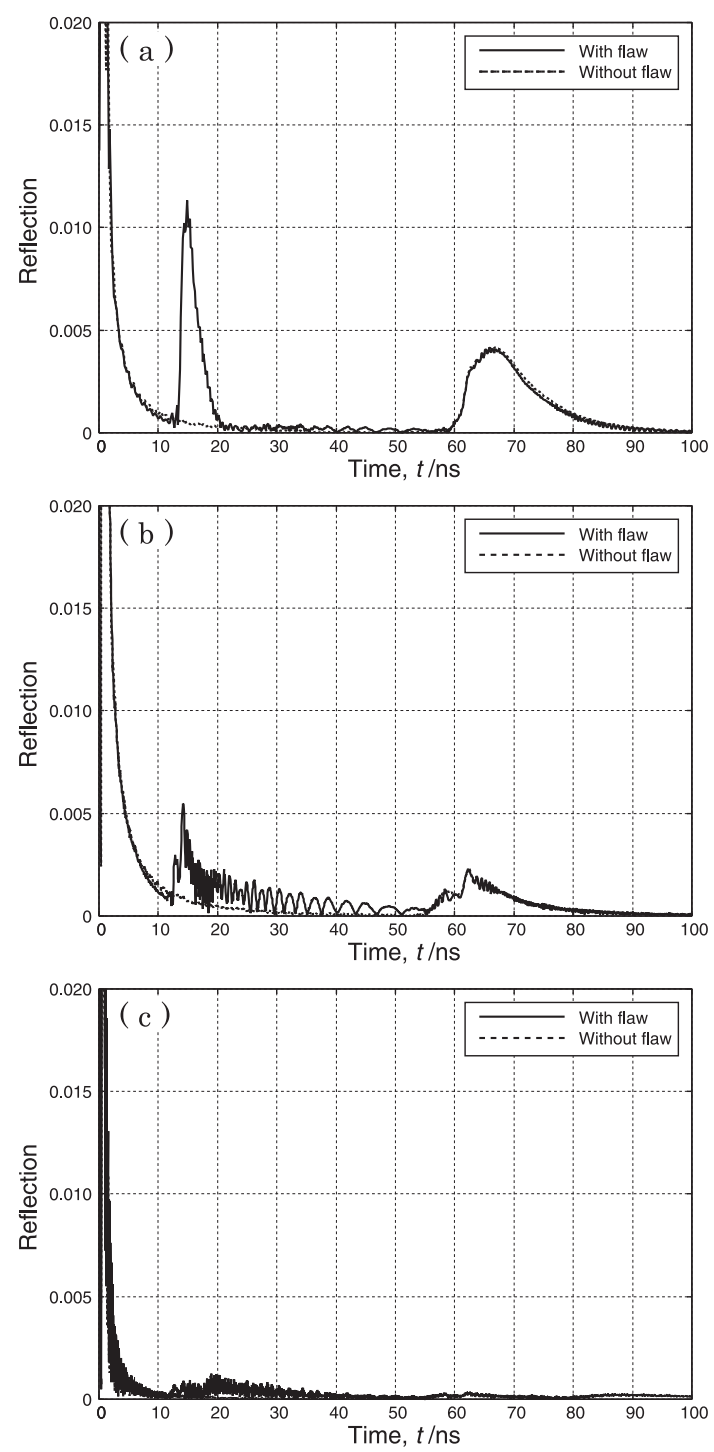

Fig. 4 The effect of frequency range on the signals due to a tube with a flaw situated $1.5 \mathrm{~m}$ away from the probe and those due to a tube without flaw. (a) 11-17 GHz, (b) 10-22.9 GHz, (c) 10-40 GHz. 
inside the tube for each mode, as evaluated by the three-dimensional finite element simulations. For example, at $15 \mathrm{GHz}$, almost $80 \%$ of microwaves propagating in the flexible cable in TEM mode were transformed into microwaves propagating inside the tube in $\mathrm{TM}_{01}$ mode, with the rest reflected at the probe; at $22 \mathrm{GHz}$, almost all microwaves were reflected at the probe.

\section{Results and Discussions}

Figure 4 presents the effect of frequency range on signals. The figure shows the time domain signals from a tube with a flaw $24.8 \mathrm{~mm}$ in length and $1.5 \mathrm{~m}$ away from the probe as well as the signals from an unflawed tube. Three frequency ranges were considered: (a) $11-17 \mathrm{GHz}$ where microwaves propagate dominantly in $\mathrm{TM}_{01}$ mode according to Fig. 3(b); (b) $10-22.9 \mathrm{GHz}$, between the cut-off frequencies of the $\mathrm{TM}_{01}$ and $\mathrm{TM}_{02}$ modes; (c) $10-40 \mathrm{GHz}$ containing all frequencies used in the experiments. The large reflections near $0 \mathrm{~ns}$ indicate reflections at the probe; those around $15 \mathrm{~ns}$ were caused by the slit. The figure shows that the 11-17 GHz range provides the clearest reflections due to the slit, although this was the narrowest of the three frequency ranges. This indicates that frequency range should be determined by the characteristics of the probe rather than cut-off frequencies.

Figure 5 presents the signals from the same tubes when their ends were closed with the metallic plate. The frequency range used to obtain the time domain signals was 11-17 GHz. Closing the tube end increased reflections after $60 \mathrm{~ns}$. This result confirms that the reflections were caused by the tube end, and corresponds with the approximate velocity $(2.2 \times$ $10^{8} \mathrm{~m} / \mathrm{s}$ ) of the microwaves inside the tube at $15 \mathrm{GHz}$ where the ratio of energies transmitted into the tube in the $\mathrm{TM}_{01}$ mode was maximized.

Figure 6 shows the signals due to flaws of different lengths and locations. The ends of the tubes were open to air, and the

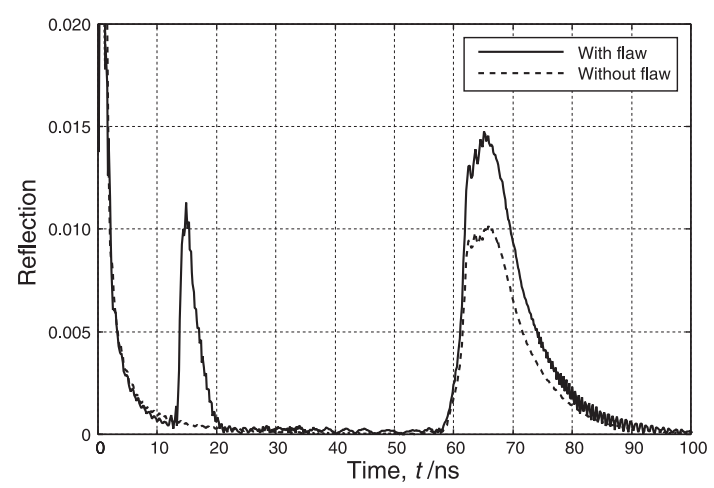

Fig. 5 Signals due to a tube with a flaw situated $1.5 \mathrm{~m}$ away from the probe and those due to a tube without flaw when the end of the tube was closed.
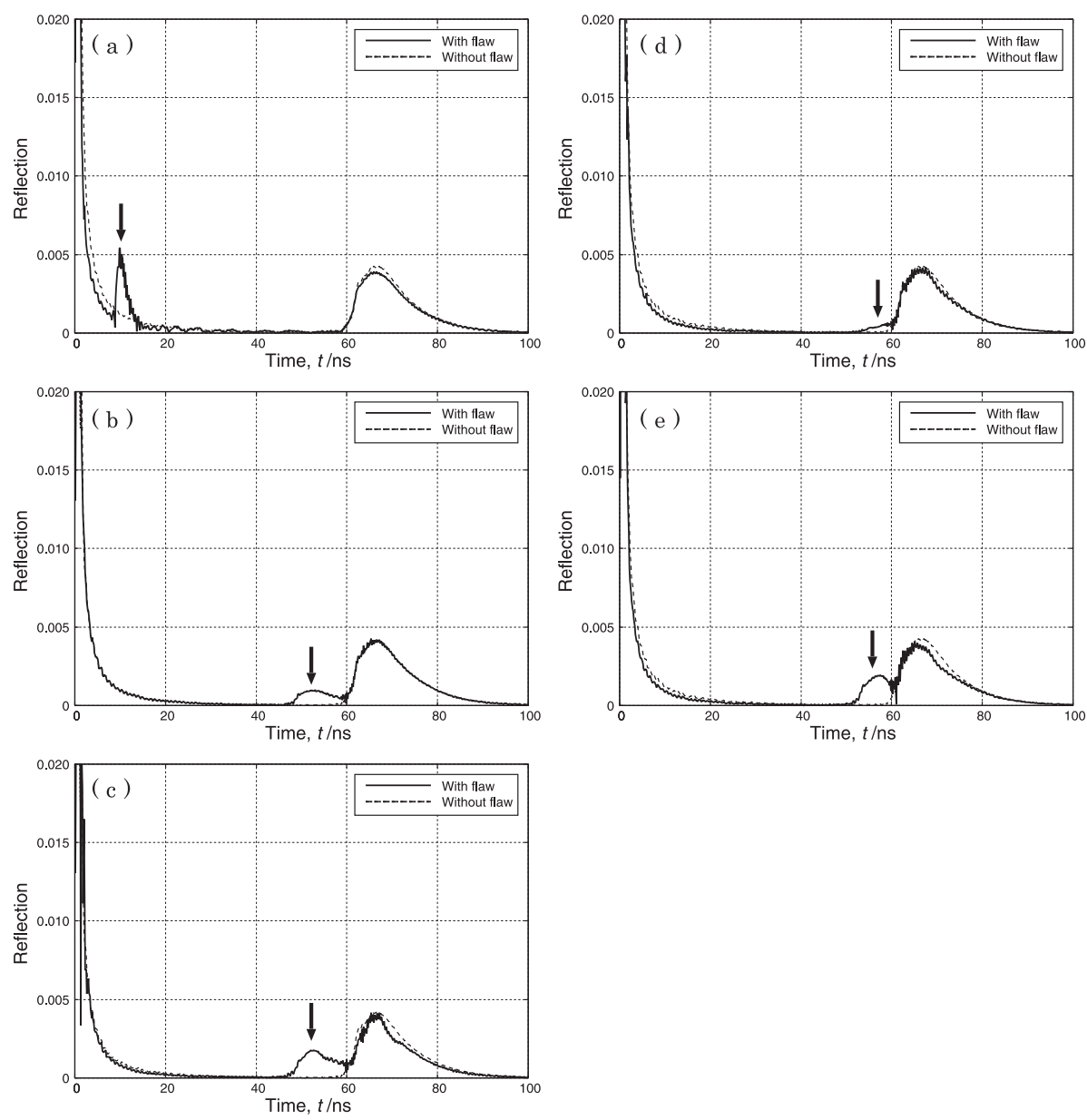

Fig. 6 Signals due to a tube containing a flaw with a distance from the probe of $\mathrm{L}$ and a length of $\mathrm{h}$. (a) $L=1.0 \mathrm{~m}, h=14.65 \mathrm{~mm}$, (b) $L=5.5 \mathrm{~m}, h=15.30 \mathrm{~mm}$, (c) $L=5.5 \mathrm{~m}, h=24.80 \mathrm{~mm}$, (d) $L=6.0 \mathrm{~m}, h=14.65 \mathrm{~mm}$, (e) $L=6.0 \mathrm{~m}, h=23.90 \mathrm{~mm}$. 

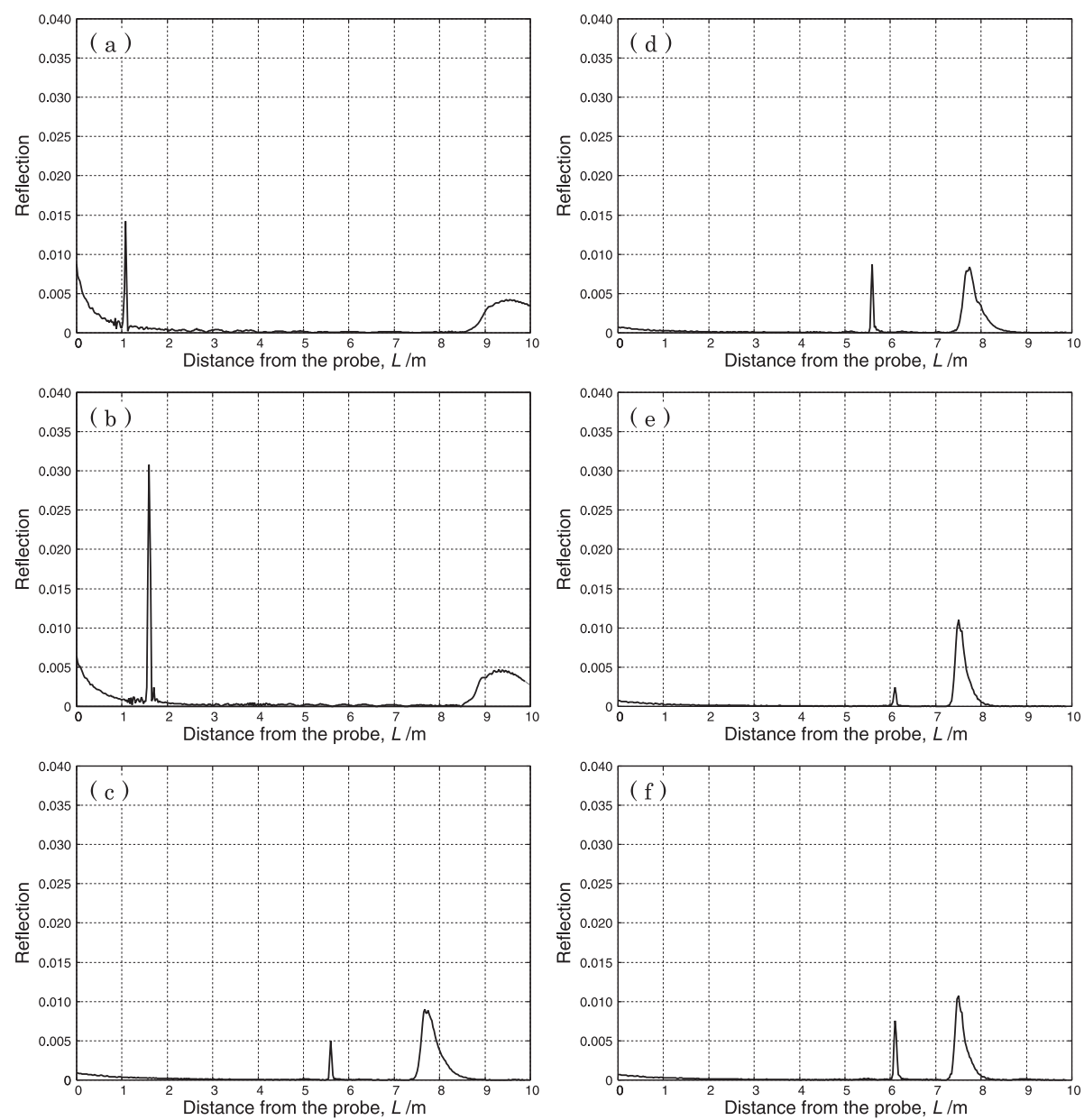

Fig. 7 Results of imposing signal processing to compensate the dispersion of microwave on the signals shown in Fig. 6 . The distance between a flaw and the probe and the length of the flaw are denoted as $\mathrm{L}$ and h, respectively. (a) $L=1.0 \mathrm{~m}, h=14.65 \mathrm{~mm}$, (b) $L=1.5 \mathrm{~m}, h=24.80 \mathrm{~mm}$, (c) $L=5.5 \mathrm{~m}, h=15.30 \mathrm{~mm}$, (d) $L=5.5 \mathrm{~m}, h=24.80 \mathrm{~mm}$, (e) $L=6.0 \mathrm{~m}, h=14.65 \mathrm{~mm}$, (f) $L=6.0 \mathrm{~m}, h=23.90 \mathrm{~mm}$.

frequency range used was 11-17 GHz. Each figure shows the signals obtained before flaw fabrication to clarify reflections due to the flaw. The figure confirms clear differences between the signals of unflawed tubes and with flaws. The locations of the differences qualitatively correspond to the locations of the flaws, whereas the dispersion of the microwave makes reflections away from the probe rather unclear. It is reasonable that a longer flaw causes a larger reflection than does a shorter flaw situated at the same location.

Figure 7 presents the results of imposing signal processing, as discussed in previous work ${ }^{7}$, on the original signals shown in Fig. 6 to compensate for the dispersion of microwaves. The reflections became markedly clearer due to the signal processing, and the locations of the reflections agreed well with the actual ones.

\section{Conclusion}

This study evaluated inner-surface cracks could be detected in a long metallic tube based on the reflections of microwaves propagating inside the tube. Because reflections due to cracks are much smaller than those due to wall thinning that have been the main focus of earlier studies, two seamless straight tubes $7 \mathrm{~m}$ in length were prepared to avoid effects of tube connections that cause reflections as large as those due to cracks. The probe to emit microwave was designed using three-dimensional finite element simulations and placed at the end of the tube, and artificial circumferential slits penetrating the tube wall were used to simulate cracks. The experiments confirmed that clear reflections could be detected from a slit $6 \mathrm{~m}$ from the probe. Furthermore, signal processing to compensate for the dispersion of microwaves propagating inside the tube both clarified the reflections and allowed for the quantitative evaluation of slit location.

\section{Acknowledgments}

The authors deeply thank Dr. Takashi Wakai of the Japan Atomic Energy Agency for his kind assistance in conducting the experiment. This study was supported by a Grant-in-Aid for JSPS Fellows and JSPS KAKENHI Number 15K14298.

\section{REFERENCES}

1) K. Sugawara, H. Hashizume and S. Kitajima: JSAEM Studies in Applied Electromagnetics and Mechanics 10 (2001) 313-316.

2) H. Hashizume, T. Shibata and K. Yuki: International Journal of Applied Electromagnetics and Mechanics 20 (2004) 171-178.

3) R.E. Jones, F. Simonetti, M.J.S. Lowe and I.P. Bradley: Journal of Nondestructive Evaluation 31 (2012) 65-76.

4) Y. Ju, L. Liu and M. Ishikawa: Mater. Sci. Forum 614 (2009) 111-116. 
5) N. Saber, Y. Ju, H.Y. Hsu and S.H. Lee: Int. J. Press. Vessels Piping 111-112 (2013) 99-105.

6) I. Nishiguchi, F. Inada, M. Takahashi, B. Ogawa, T. Inagaki, T. Ohira, K. Iwahara and K. Yamakami: E-Journal of Advanced Maintenance 2 (2010) 14-24.

7) Y. Sakai, N. Yusa and H. Hashizume: Nondestructive Testing and Evaluation 27 (2012) 171-184.

8) K. Sasaki, L. Liu, N. Yusa and H. Hashizume: NDT\&E Int. 70 (2015)
53-59.

9) K. Sasaki, N. Yusa and H. Hashizume: International Conference on Maintenance Science and Technology, Kobe, Japan, 2014/11/02-05.

10) T. Onizawa, Y. Nagae, S. Takaya and T. Asayama: Proceedings of the ASME 2013 Pressure Vessels and Piping Conference, PVP2013-97611, Paris, France, 2016/07/14-18.

11) K. Aoto, N. Uto, Y. Sakamoto, T. Ito, M. Toda and S. Kotake: J. Nucl. Sci. Technol. 48 (2011) 463-471. 